\title{
AfsR is an important regulatory factor for growth and butenyl-spinosyn biosynthesis of Saccharopolyspora pogona
}

\section{$\mathrm{Li} \mathrm{Li}^{1}$ • Liang Gong ${ }^{1} \cdot$ Haocheng He ${ }^{1}$. Zhudong Liu ${ }^{1}$. Jie Rang ${ }^{1} \cdot$ Jianli Tang ${ }^{1} \cdot$ Shengnan Peng ${ }^{1}$. Shuangqin Yuan ${ }^{1}$. Xuezhi Ding ${ }^{1} \cdot$ Ziquan $\mathrm{Yu}^{1} \cdot$ Liqiu Xia $^{1} \cdot$ Yunjun Sun ${ }^{1}$}

Received: 22 September 2018 / Accepted: 12 April 2019 / Published online: 26 April 2019

(C) Università degli studi di Milano 2019

\begin{abstract}
Purpose To generate a AfsR-like (AfsR-L) overexpression strain Saccharopolyspora pogona-AfsR-L and investigate its effects on the morphology and metabolism of S. pogona.

Methods Firstly, we generated the overexpression vector pOJ260- $P_{\text {erme }}-a f s R-L$ via overlap extension PCR. Then, the recombination strain $S$. pogona-AfsR-L was constructed via conjugal transfer. To monitor the growth and morphology, mycelia and sporulation were observed. The distinctive proteins and butenyl-spinosyn biosynthesis were investigated by SDS-PAGE, HPLC, and mass spectrometry. And the transcriptional level of afsR-L and other relative functional genes in $S$. pogona-AfsR-L was analyzed by qRT-PCR. Western blot verified the increased amount of AfsR-L protein in the overexpression strain.

Result Growth curve and mycelia observation showed that afsR-L overexpression make the stationary phase of $S$. pogona-AfsRL longer than that of wild $S$. pogona by approximate 3 days. Moreover, S. pogona-AfsR-L exhibited a more obvious white phenotype on the solid medium, which means afsR-L overexpression affects the sporulation ability of $S$. pogona. HPLC analysis revealed that the peak area of the butenyl-spinosyn yield of $S$. pogona-AfsR-L was 293.6, while that of $S$. pogona was 250.9. SDS-PAGE analysis showed that the two strains had different whole protein expression profiles, and the distinctive proteins were further identified by LC-MS/MS identification, which showed the possible control mechanism of afsR-L gene in S. pogona. Conclusion We concluded that AfsR could directly or indirectly positively regulate the biosynthesis of butenyl-spinosyn and affect the growth features of S. pogona. We envisioned that this result can be expanded to other Streptomyces for strain improvement.
\end{abstract}

Keywords Saccharopolyspora pogona $\cdot$ AfsR $\cdot$ Regulatory factor $\cdot$ Butenyl-spinosyn biosynthesis $\cdot$ Strain morphology

\section{Introduction}

Butenyl-spinosyn is a macrolide secondary metabolite that is produced by S. pogona (Hahn et al. 2006; Lewer et al. 2009). This metabolite depolarizes insect nerve cells by binding to nicotinic acetylcholine receptors, thereby inducing the hyperactivity

$\mathrm{Li} \mathrm{Li}$ and Liang Gong contributed equally to this work.

Electronic supplementary material The online version of this article (https://doi.org/10.1007/s13213-019-01473-8) contains supplementary material, which is available to authorized users.

Yunjun Sun

sunyj@hunnu.edu.cn

1 State Key Laboratory of Development Biology of Freshwater Fish, Hunan Provincial Key Laboratory for Microbial Molecular Biology, College of Life Science, Hunan Normal University, Changsha, China of the central nervous system that causes the rapid death of insects (Millar and Denholm 2007). Butenyl-spinosyn has extensive insecticidal activity against a wide spectrum of insect pests, including Lepidoptera, Diptera, and Coleoptera (Kirst 2010; Millar and Denholm 2007). However, wild S. pogona has very low capability for the biosynthesis of butenyl-spinosyn (Lewer et al. 2009; Li et al. 2018). Therefore, enhancing its biosynthesis is crucial for its wide application in the biocontrol of insect pests. Genetic engineering technology is a common and effective means for strain improvement. Liu reported that AtEDT1 overexpression in Salvia miltiorrhiza significantly increases salvianolic acid yield (Liu et al. 2017). Sun found that knocking out the negative control gene sav_742 in Streptomyces avermitilis increased abamectin production, compared with that of wild strain (Sun et al. 2016). Zhu utilized genetic engineering techniques to overexpress the $\sin R$ gene in Streptomyces albus and found that salinomycin yield is $25 \%$ higher than that of wild 
strain (Zhu et al. 2017). Zhang reported that overexpressing the milR gene of Streptomyces bingchenggensis increases milbemycin A3/A4 production by $38 \%$ (Zhang et al. 2016). These studies showed that the use of genetic engineering technology to knock out or overexpress the genes related to the secondary metabolism in Streptomyces could effectively promote the biosynthesis of target secondary metabolites.

The global regulatory gene, not only participating in various life activities of organisms, but also involving in secondary metabolites biosynthesis, is located outside the biosynthetic gene cluster of secondary metabolites and regulates the expression of corresponding pathway-specific genes (Tatarko and Romeo 2001). Compared with other regulatory genes, the global regulatory gene is a more diverse, universal, and complex regulatory element of secondary metabolism in Streptomyces (Horinouehi et al. 1990; Xu et al. 2017).

AfsR is a global regulatory factor present in Streptomyces, which belongs to the Streptomyces antibiotic regulatory protein (SARP) family encoded by the $a f s R$ gene (Tanaka et al., 2007 ). The function of afs $R$ gene has been extensively studied in Streptomyces coelicolor, which could activate the transcription of the afsS gene, regulate the biosynthesis of actinorhodin and undecylprodigiosin. AfsR protein, due to two domains containing ATP binding and DNA-binding domains, has an important influence on pigment phenotype of $S$. coelicolor. Furthermore, the DNA binding and ATPase activities of AfsR are physically separable, and these two functions are regulated by phosphorylation on serine and threonine residues (Sueharu Horinouehi, et al. 1990; Tanaka et al., 2007; PingChin Lee, et al. 2002). Fernando Santos-Beneit found that AfsR and PhoP regulate the biosynthesis of actinorhodin and undecylprodigiosin by controlling the $a f s S$, pstS, and phoRP transcription, PhoP may downregulate afs $S$ expression by competitively binding to its promoter, and AfsR also competes with PhoP for binding to the promoters of $p s t S$ and phoRP (Fernando Santos-Beneit, et al. 2009).

AfsR regulates the biosynthesis of multiple secondary metabolites and activates different antibiotic biosynthetic gene clusters via different mechanisms. The afs $R-P$ gene from Streptomyces peucetius ATCC 27952 had 60\% similarity to that from $S$. coelicolor. When placing this gene under the strong promoter of $P_{\text {ermE }}$, the yield of $\gamma$-actinorhodin and clavulanic acid in Streptomyces clavuligerus increased by $260 \%$ and $150 \%$, respectively (Parajuli, et al. 2005). Wang reported that afs $R$ gene overexpression in Streptomyces lomondensis increases lorcomycin yield by 2.5 -fold (Wang, et al. 2015). Sushila found that the overexpression of $a f s R$ $\mathrm{sp}$ in Streptomyces venezuelae stimulated the expression of the pathway-specific regulatory genes, pikD and ketosynthase, which caused the production of pikromycin was increased by 2.6-fold (Maharjan, et al. 2008).

AfsR-L (orf06333-4034) protein in S. pogona shares $57 \%$ sequence similarity to AfsR in Streptomyces $s p$.
NRRL WC-3742 (GenBank accession no. WP 078911560.1) (Supplementary Fig. 1). To study the regulation of afs $R-L$ gene in $S$. pogona, it was overexpressed under the strong promoter of $P_{\text {ermE }}$. The effects of overexpression on the strain growth and secondary metabolite biosynthesis were also investigated.

\section{Materials and methods}

\section{Bacterial strains, plasmids, and growth conditions}

The bacterial strains, plasmids, and primers used in this study are presented in Supplementary Table 1. The spores of $S$. pogona NRRL 30141 were inoculated in activation broth (glucose, $10 \mathrm{~g} / \mathrm{L}$; tryptic soy broth, $45 \mathrm{~g} / \mathrm{L}$; yeast extract, $9 \mathrm{~g} / \mathrm{L} ; \mathrm{MgSO}_{4} \cdot 7 \mathrm{H}_{2} \mathrm{O}, 2.2 \mathrm{~g} / \mathrm{L}$ ) and cultivated at $30{ }^{\circ} \mathrm{C}$ with $300 \mathrm{rpm}$ for $48 \mathrm{~h}$, then $3 \mathrm{~mL}$ bacteria suspension was transferred to $30 \mathrm{~mL}$ fermentation broth $\left(\mathrm{KNO}_{3}, 1 \mathrm{~g} / \mathrm{L} ; \mathrm{FeSO}_{4}, 10 \mathrm{mg} / \mathrm{L} ; \mathrm{K}_{2} \mathrm{HPO}_{4} \cdot 3 \mathrm{H}_{2} \mathrm{O}, 500 \mathrm{mg} /\right.$ $\mathrm{L} ; \mathrm{MgSO}_{4} \cdot 7 \mathrm{H}_{2} \mathrm{O}, 500 \mathrm{mg} / \mathrm{L}$; glucose, $20 \mathrm{~g} / \mathrm{L}$; yeast extract, $4 \mathrm{~g} / \mathrm{L}$; tryptone, $4 \mathrm{~g} / \mathrm{L} ; \mathrm{pH}, 7.2)$ and incubated at $30{ }^{\circ} \mathrm{C}$ with $300 \mathrm{rpm}$. The medium for the $S$. pogonaAfsR-L was the same as the wild strain and contained antibiotic of apramycin at $50 \mu \mathrm{g} / \mathrm{mL}$ ). All E. coli strains were grown in $\mathrm{LB}$ broth at $37^{\circ} \mathrm{C}$. When necessary, the growth medium was supplemented with antibiotic (apramycin, $50 \mu \mathrm{g} / \mathrm{mL}$ ).

\section{Determination of afsR gene homologous sequences}

The whole genome sequencing of S. pogona NRRL 30141 was completed by the third-generation genome sequencing technique (GenBank accession no. CP031142). AfsR-L protein was found from the sequencing results, and then the sequence homology analysis was performed for this protein by BLAST (https://blast.ncbi.nlm.nih.gov/Blast.cgi). It was found that its homology with AfsR in Streptomyces $s p$. NRRL WC-3742 was 57\%, and named as AfsR-L.

\section{Construction of afs $R$ - $L$ overexpression recombinant strains}

$P_{\text {ermE }}$ is a strong promoter commonly used to initiate gene expression in the Streptomyces (Luo, et al. 2015). The strong constitutive promoter $P_{\text {ermE }}$ was amplified from plasmid pOJ260-cm- $P_{\text {ermE }}$ (Li, et al. 2018) using primers $P_{\text {ermE }}-\mathrm{F}$ and $P_{\text {erme }}-\mathrm{R}$ (Supplementary Table 1 ), and the afsR-L gene was amplified using primers $a f_{S} R-L-\mathrm{F}$ and $a f_{s} R-L-\mathrm{R}$ from genomic DNA of $S$. pogona NRRL 30141 as template. Then, the two PCR fragments were fused by overlap extension PCR using primers $P_{\text {ermE }}-\mathrm{F}$ and $a f_{S} R-L-\mathrm{R}$. The fusion fragment was excised using $X b a \mathrm{I}$ and $E c o \mathrm{RV}$, and cloned into the 
Fig. 1 Identification of recombinant strain $S$. pogonaAfsR-L. a PCR amplification of aac(3) IV gene (M, DNA marker; 1 , PCR products of S. pogonaAfsR-L; 2, negative control S. pogona genome as template). b PCR detection of the fusion fragment $P_{e r m E^{-}} a f_{S} R-L$ (M, DNA Marker; 1 , positive control using pOJ260- $P_{\text {erme }}-a f_{S} R-L$ as template; 2 , PCR products of S. pogona-AfsR-L; 3, negative control using $S$. pogona genome as template). c qRT-PCR results of $a f s R-L$ transcriptional level differences between the wild type and the engineering strain. $\mathbf{d}$ Western blot results of AfsR-L expression level differences between the wild type and the engineering strain
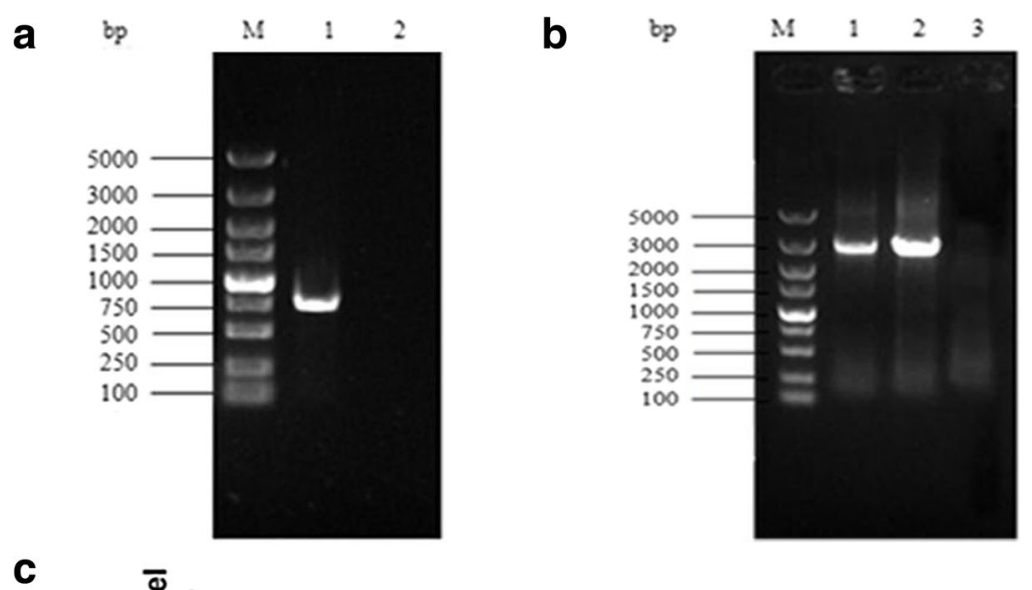

C

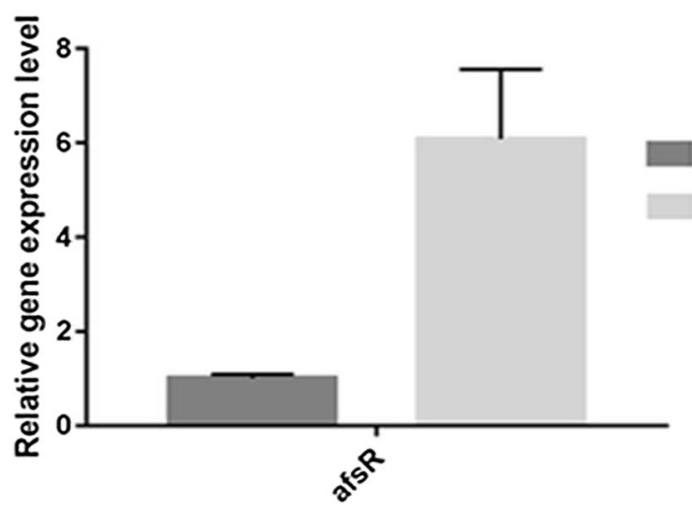

S.pogona

S.pogona-AfsR-L

d

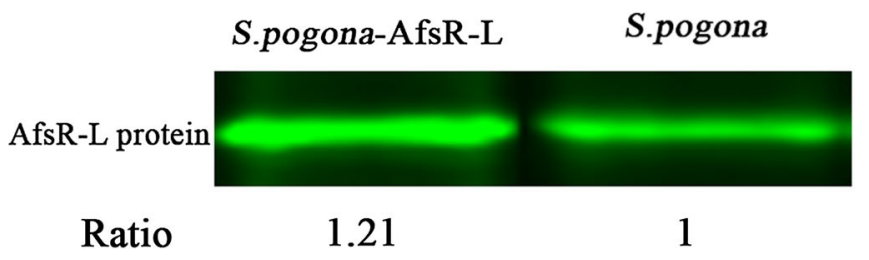

corresponding restriction sites of pOJ260 plasmid, producing the recombinant pOJ260-PermE-afs $R-L$ plasmid (Supplementary Fig. 2). This recombinant plasmid was transferred into $S$. pogona by standard conjugation methods (Tang, et al. 2011) and integrated into the genome by singlecrossover homologous recombination with afs $R-L$ gene as homology region, yielding recombinant strain $S$. pogona-AfsR-

L (Supplementary Fig. 3).

To confirm the recombinant strain, S. pogona-AfsR-L was constructed successfully, chromosomal DNA from the wild type and recombinant strains was isolated, the inserted gene was analyzed by PCR amplification, and its expression was further analyzed by qRT-PCR and Western blot.

\section{Cultivation profile analysis of the wild type and recombinant strains}

To monitor the growth profiles, the growth curve of wild type and recombinant strains was determined through measuring optical density of fermentation broth $\left(\mathrm{OD}_{600}\right)$ every
$12 \mathrm{~h}\left(30^{\circ} \mathrm{C}, 300 \mathrm{rpm}\right)$. They were performed in triplicate. After cultivation for 2 days, $40 \mu \mathrm{L}$ broth of wild type and recombinant strains was spread on BHI, R6, and TSB solid medium and incubated at $30{ }^{\circ} \mathrm{C}$. The same amount of cultured strains was inoculated into $20 \mathrm{~mL}$ TSB medium culture for 2 days. Then, Hitachi SU8000 cold field emission scanning electron microscope (SEM) was used to observe the cell morphology. After fermentation for 10 days, the quantification of butenyl-spinosyn yield by UV-HPLC was performed as previously described with slight modification (Yang, et al. 2014). Butenyl-spinosyn was extracted by incubating the cell-free supernatant with acetone at a 1:1 volume ratio for $48 \mathrm{~h}$. Cultures were centrifuged at $9000 \mathrm{rpm}$ for $15 \mathrm{~min}$, and the supernatants were filtered through 0.22- $\mu \mathrm{m}$ Millipore filters. After filtration, a $10 \mu \mathrm{L}$ aliquot of each supernatant was loaded onto a $\mathrm{C} 18$ column (AQ12S05-1546WT) and eluted with the elution buffer at $1.0 \mathrm{~mL} / \mathrm{min}$. The elution buffer contained methanol, acetonitrile $(\mathrm{ACN})$, and $2 \%$ aqueous ammonium acetate in a specific volumetric ratio $(1: 1)$. The detection wavelength was set at $250 \mathrm{~nm}$ during the analysis. 


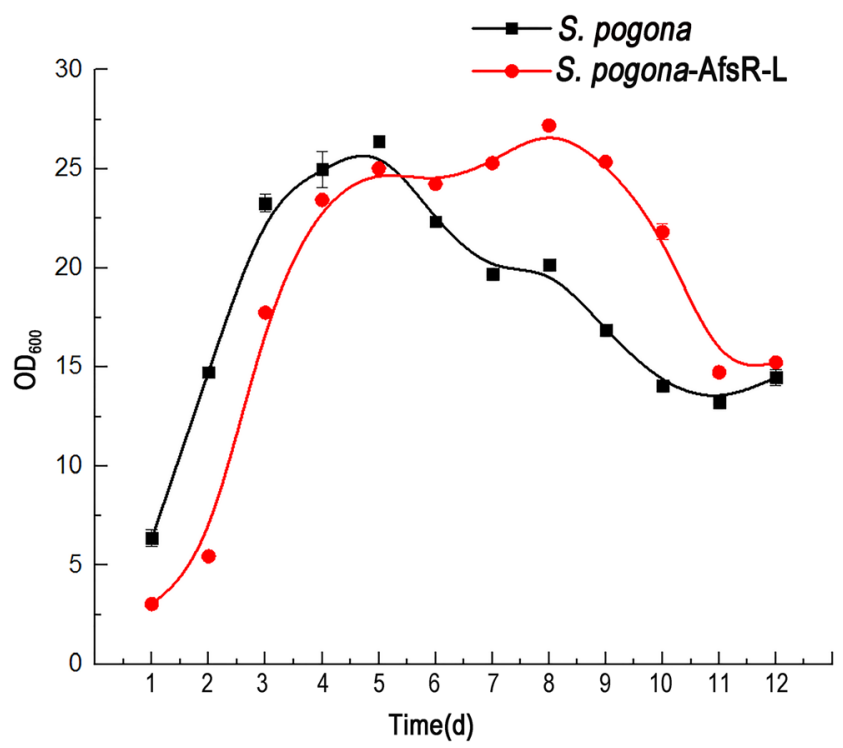

Fig. 2 Growth curve of S. pogona-AfsR-L and S. pogona. At the initial time, growth of $S$. pogona and $S$. pogona-AfsR-L was basically synchronized and the two strains entered stationary phase at 4 days. But as the time went by, the stationary phase of the wild-type strain S. pogona was short and the decline phase commenced at 5.5 days, whereas the engineered strain $S$. pogona-AfsR-L entered decline phase at 8 days. The highest biomass for $S$. pogona-AfsR-L was also higher than that for the wild-type strain S. Pogona

\section{Protein extraction and SDS-PAGE analysis}

To analyze the difference of the whole-cell protein between the wild type and recombinant strains, cells were harvested after 5 days and washed for four times with PBS $(10 \mathrm{mM}$, $\mathrm{pH} 7.8$, prechilled at $4{ }^{\circ} \mathrm{C}$ ), resuspended in $200 \mu \mathrm{L}$ of lysozyme $(100 \mathrm{mg} / \mathrm{mL})$ for $1 \mathrm{~h}$, added with $600 \mu \mathrm{L}$ lysis buffer (8 M urea; $2 \mathrm{M}$ thiourea; $4 \%$ CHAPS; $75 \mathrm{mM} \mathrm{NaCl} ; 50 \mathrm{mM}$ Tris-HCl, pH: 8; $2 \mathrm{mM}$ phenylmethylsul-fonyl fluoride; $4 \mu \mathrm{L}$ of a protease inhibitor cocktail powder: P8465, Sigma, St. Louis, MO, USA) in each tube, and ultrasonically fragmented (JY92-II ultrasonic cell grinder, Ningbo Scientz biotechnology CO., LTD). After the quantitative analysis of the protein by Bradford assay, the components of protein samples were checked by SDS-PAGE.

\section{Total RNA isolation and qRT-PCR analysis}

To perform transcriptional analysis, total RNA from the wild type and recombinant strain was separately isolated following instructions of Total RNA Extractor Kit (Sangon Biotech) with slight modifications, and each strain was performed in triplicate. Briefly, bacteria, after incubated 5 days, were harvested from $1 \mathrm{~mL}$ suspension at $8000 \mathrm{rpm}$ for $2 \mathrm{~min}$, and immediately frozen in liquid nitrogen. Next, it was ground in liquid nitrogen and homogenized in $1 \mathrm{~mL}$ Trizol. The solution was mixed with $0.25 \mathrm{~mL}$ chloroform followed by an ice bath for $5 \mathrm{~min}$. After centrifugation at $12000 \mathrm{rpm}, 4{ }^{\circ} \mathrm{C}$ for
$10 \mathrm{~min}$, the aqueous phase was transferred to a new $1.5-\mathrm{mL}$ tube and added equal volume of isopropanol with subsequent ice bath for $1 \mathrm{~h}$. The mixture was centrifuged, and then the precipitation was washed twice with $75 \%$ ethanol and dissolved in RNase free water. RNA concentration and purity were established by measuring the ratio of $\mathrm{OD}_{260} \mathrm{~nm}$ to $\mathrm{OD}_{280} \mathrm{~nm}$. DNase treatment and cDNA synthesis were performed by PrimeScript ${ }^{\mathrm{TM}}$ RT Reagent Kit with gDNA Eraser (Takara) according to the manufacturer's instructions. The real-time qPCR amplification was performed on 7500 RealTime PCR system instruments (Applied Biosystems, USA). The 16S rRNA gene was used as an internal control to quantify the relative expression of target genes.

\section{Heterologous expression and Western blot analysis of AfsR protein}

The $a f_{s} R$ gene was amplified using primers $a f_{s} R-L-\mathrm{F}$ (WB) and afs $R-L-\mathrm{R}(\mathrm{WB})$ from genomic DNA of $S$. pogona (Supplementary Table 1). The amplified $a f s R$ gene was cloned into pET-28a using an inNovafast Assembly Cloning Kit (Innovagene), and then transferred to E. coli BL21 (DE3). The recombinant strain was inoculated into LB medium supplemented with $40 \mu \mathrm{g} / \mathrm{mL}$ kanamycin. After induced with $0.5 \mathrm{mM}$ isopropyl- $\beta$-D-thiogalactopyranoside (IPTG), the cells were harvested by centrifugation and resuspended in PBS buffer $(137 \mathrm{mM} \mathrm{NaCl}, 2.7 \mathrm{mM} \mathrm{KCl}, 10 \mathrm{mM}$ $\mathrm{Na}_{2} \mathrm{HPO}_{4}, 1.8 \mathrm{mM} \mathrm{KHPO}_{4}$ ). The cells were disrupted by sonication. Subsequently, the inclusion body was collected through centrifugation and the AfsR protein was purified by a Ni-NTA column. Anti-AfsR-L antibody was obtained through immunizing rabbit (Supplementary Table 2) as describe previously (Mikael, et al. 2017).

Twelve microgram of protein samples derived from S. pogona and S. pogona-AfsR-L were separated by SDSPAGE gel and transferred to PVDF membrane. The membrane was incubated with primary antibody and then the secondary antibody (IRDye 680 Conjugated Goat Anti-Rabbit IgG, 1:10000). Odyssey infrared fluorescence detection system (Li-COR Biosciences, Lincoln, NE) was used to detect the results (Yang, et al. 2014; Stefanie Muller, et al. 2007).

\section{Nano-LC-MS/MS analysis}

Differential protein bands between the wild type and recombinant strains were cut out of the SDS-PAGE gel for in-gel tryptic digestion and proceed with liquid chromatographytandem mass spectrometry (LC-MS/MS) analysis (Luo, et al. 2011, Yang, et al. 2014). 1D-LC-MS/MS analysis was performed as described previously (Huang, et al. 2012) by using LTQ XL mass spectrometer (Thermo Fisher, San Jose, CA, USA). 


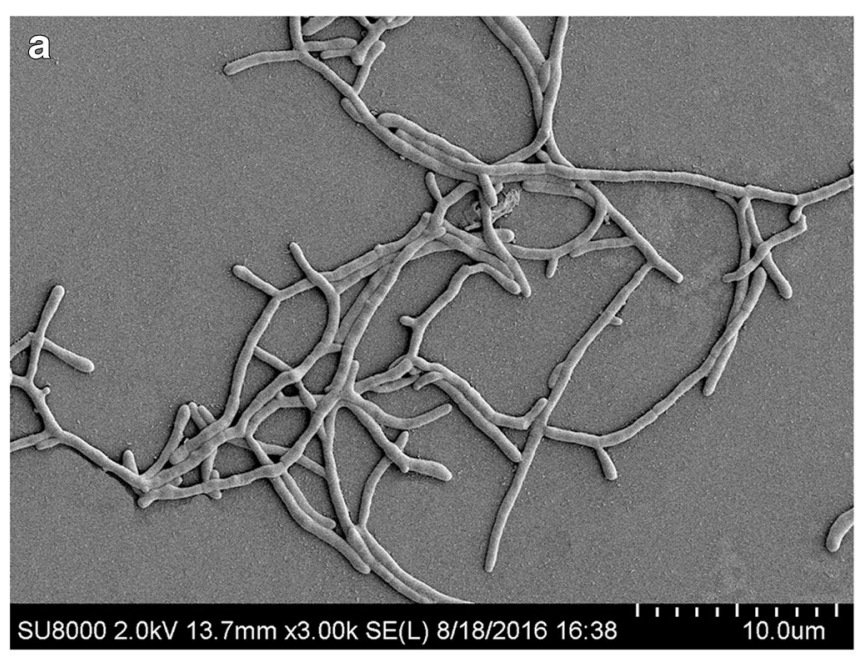

C

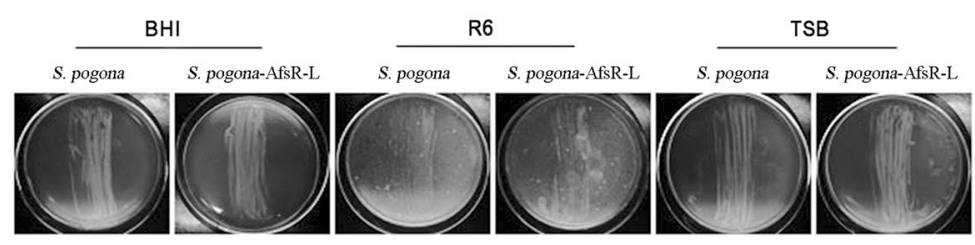

3 day
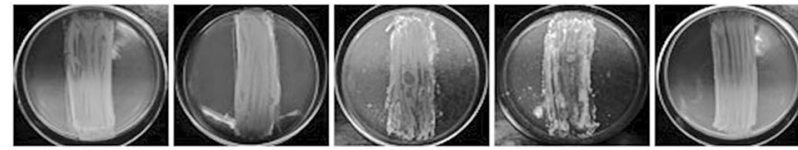

6 day
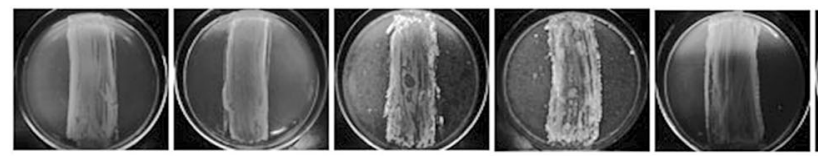

Fig. 3 The morphological comparison of wild type and the engineered strains. a Scanning electron microscopic observation of $S$. pogona. b Scanning electron microscopic observation of $S$. pogona-AfsR-L. The mycelia of $S$. pogona-AfsR-L grew faster than those of $S$. pogona. Its hyphae were more branched and developed than those of S. pogona. Bars, $10 \mu \mathrm{m}$. $\mathbf{c}$ The comparison of spore formation on different solid media. The two strains were grown on the three solid media plate and observed at days 1, 3, and 6 after inoculation. Spores were produced after 3 days on the three solid media. The sporulation ability of the two strains on BHI

\section{Results}

\section{Effects of afs $R$ - $L$ overexpression on the growth of S. pogona}

S. pogona-AfsR-L strain was successfully obtained according to the proof of gene level, transcriptional level and protein expression level (Fig. 1). To find the difference between S. pogona and S. pogona-AfsR-L in fermentation broth, the growth characteristics were measured and controlled in real time (Fig. 2). The result showed that initial growths of $S$. pogona and $S$. pogona-AfsR-L were basically synchronized. The two strains entered stationary phase at 4 days (Fig. 2). However, the stationary phase of the wild-type strain

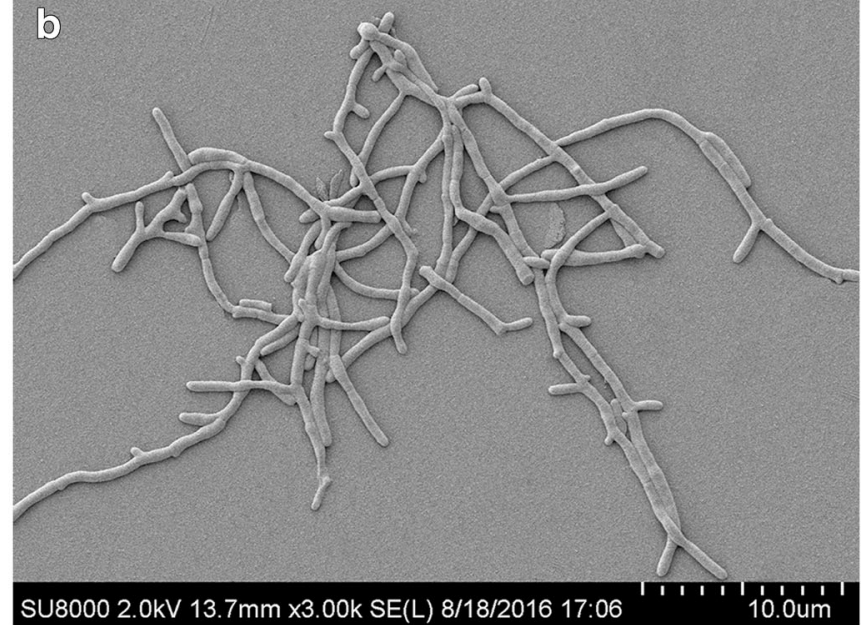

d

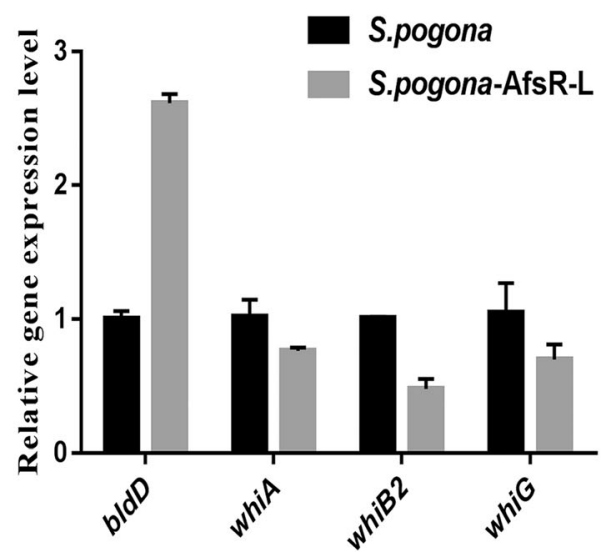

and TSB solid media was lower than on R6 solid medium. d Expression level of whiA, whiB2, whiG, and bldD. The mRNA samples were isolated after 3 days from the wild type and engineered strains. Expression of $b l d D$ exhibited a significant upregulation in the mutant, whereas whiA,whiB2, and $w h i G$ were downregulated, the $16 \mathrm{~S}$ rRNA gene was used as an internal control to quantify the relative expression of target genes. Black bars represent $S$. pogona, and gray bars represent $S$. pogona-AfsR-L. Gene expression differences were shown by bars height. Error bars represent standard deviation of the mean

S. pogona was short and the decline phase commenced at 5.5 days, whereas the engineered strain S. pogona-AfsR-L started decline phase at 8 days. In addition, the resultant biomass for S. pogona-AfsR-L was also higher than that for the wild-type strain $S$. pogona.

\section{Effects of afs $R$ - $L$ overexpression on the morphology of S. pogona}

The SEM observation of the two strains revealed that the mycelia of $S$. pogona-AfsR-L grew faster than those of $S$. pogona. Moreover, its hyphae were more branched and developed (Fig. 3a, b). To research the effect of afs $R-L$ overexpression on sporulation in different solid culture 

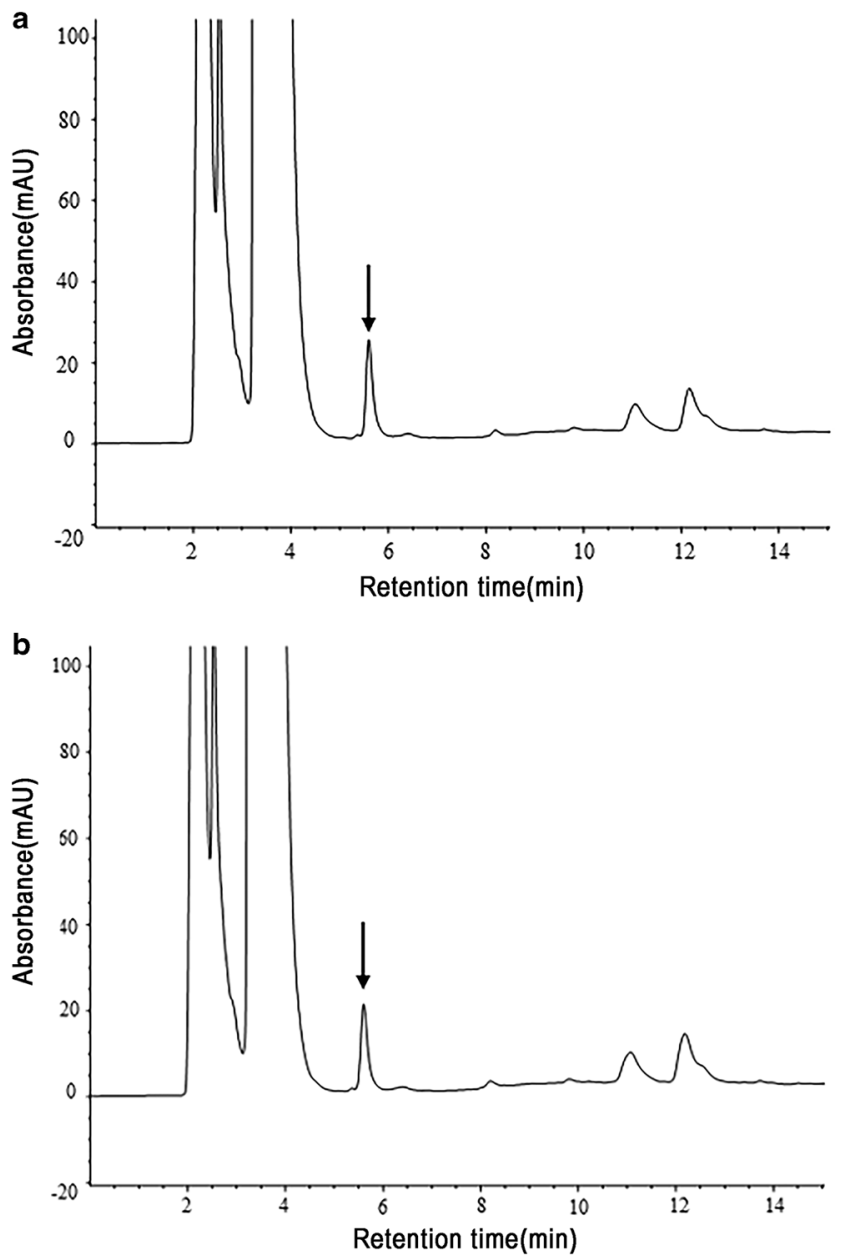

Fig. 4 The butenyl-spinosyn production analysis. a Butenyl-spinosyn production of $S$. pogona-AfsR-L. b Butenyl-spinosyn production of $S$. pogona. The peak area of the butenyl-spinosyn yield of $S$. pogonaAfsR-L and S. pogona was 293.6 and 250.9 , respectively

medium, strains of S. pogona-AfsR-L and S. pogona were observed on BHI, R6, and TSB solid medium. S. pogonaAfsR-L obviously had a white phenotype on the three media compared with $S$. pogona, especially on the R6 solid medium (Fig. 3c). To identify the different phenotypes, several whi-like (whi-L) genes and bldD gene were selected through homology analysis to check the transcriptional level, which has a significant influence on strain phenotype and sporulation by whi-L family genes negative regulation and $b l d D$ gene positive regulation (Bush, et al. 2016; Elliot, et al. 2003; Kim, et al. 2006; Virginie Molle 2000; Yoshihiro Mouri 2017). Expression of whiA, whiB2, and whiG was lower in the mutant, especially whiB2 that showed $52.2 \%$ downregulation. However, bldD gene expression level was increased by $160.5 \%$ in the mutant (Fig. 3d), which acts to repress expression of the whi genes (Bush, et al. 2016; Den Hengst, et al. 2010). These results provide an explanation for the special phenotype of the mutant.
Effects of afs $R$ - $L$ overexpression on butenyl-spinosyn biosynthesis

Butenyl-spinosyn was mainly found in the cell-free supernatant (Supplementary Fig. 4), and the extracted butenylspinosyn from the fermentation broth was subsequently examined by HPLC. The target peaks were harvested for MS identification; the results show that the $(\mathrm{M}+\mathrm{H})+$ ions at $\mathrm{m} / \mathrm{z}=$ 634 contained $617[\mathrm{M}+\mathrm{H}]+(\mathrm{m} / \mathrm{z})$ and a rhamnose ion fragment with a molecular mass of 189 (Supplementary Fig. 5). This finding proved that the substance was spinosyn $\alpha \mathrm{d}$, a butenylspinosyn component, which is consistent with previous data (Lewer, et al. 2009). The HPLC results showed that butenylspinosyn yield of $S$. pogona-AfsR-L was 293.6 and that of $S$. pogona was 250.9 . Therefore, the total concentration of butenyl-spinosys produced by $S$. pogona-AfsR-L increased to $117 \%$ compared with that of S. pogona (Fig. 4), which implies afs $R$ - $L$ gene overexpression improves the butenylspinosyn yield of the engineered strain S. pogona-AfsR-L.

\section{Analysis of whole-cell protein}

After culture of $120 \mathrm{~h}$, SDS-PAGE analysis showed that the two strains had significantly different whole protein profiles. Distinctive bands were identified by 1D-LC-MS/ MS (Fig. 5a) and their expression level was further confirmed by qRT-PCR analysis (Fig. 5b). The results revealed that the protein band A was identified as polynucleotide phosphorylase and exhibited $1000.3 \%$ increase in S. pogona-AfsR-L. The protein bands B and C decreased by $32.2 \%$ and $84.7 \%$ in S. pogona-AfsR-L and were identified as the molecular chaperone GroEL ( $g r o E L)$ and senoyl-CoA hydratase (ech), respectively. The identified proteins were then categorized using UniProt (www. uniprot.org) and Gene Ontology (GO) for functional analysis. Polynucleotide phosphorylase ( $p n p)$ is involved in mRNA degradation by function analysis, molecular chaperone GroEL prevents misfolding and promotes the refolding and proper assembly of unfolded polypeptides that are generated under stress conditions, and senoylCoA hydratase is related with the pathway of fatty acid beta-oxidation, which is part of lipid metabolism (Table 1).

\section{Discussion}

The isolated secondary metabolites of Streptomyces have important application as antibiotics, enzymes, and immunosuppressive activity. The structure and function of secondary metabolites have been continuously selected and optimized over a long-term natural evolution, resulting in a unique chemical structure with superior activity. These secondary metabolites are an important biological resource 


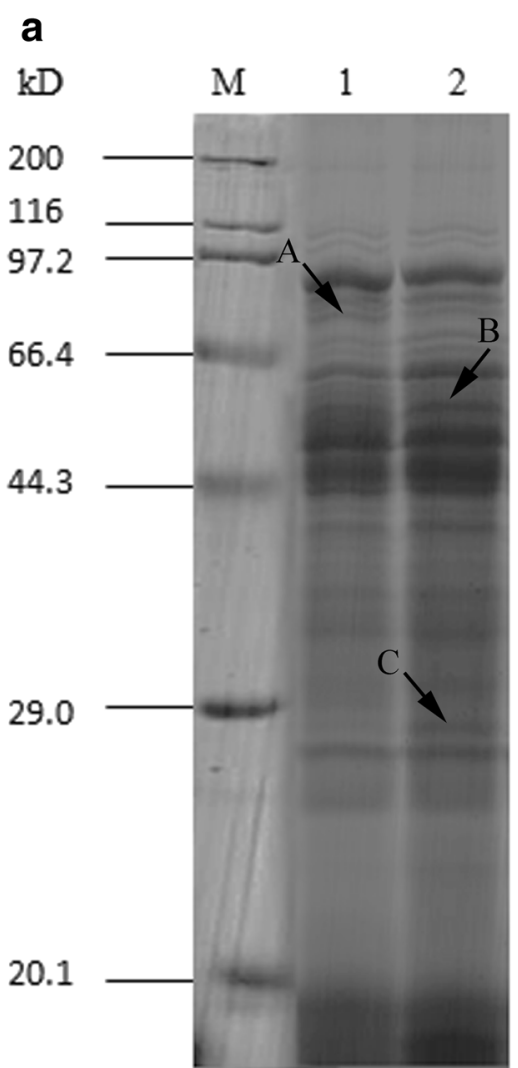

Fig. 5 Distinctive protein analysis. a SDS-PAGE gel analysis of total proteins. M, protein marker; 1 , total proteins from $S$. pogona-AfsR-L at 5 days; 2 , total proteins from $S$. pogona at 5 days. Three distinctive bands were observed between the two strains, which were identified via 1D-LCMS/MS. b qRT-PCR results of pnp, groEL, and ech transcriptional level. The mRNA samples were isolated from $S$. pogona-AfsR-L and S. pogona

for the drug research and development. The development of gene sequencing and DNA recombination technologies has enabled the elucidation of the biosynthetic pathway and regulatory mechanism of various microbial natural products. Furthermore, these technologies provide an effective method for genetic modification of the Streptomyces genome. The yield of secondary metabolites can be increased based on the specificity of a regulatory pathway, overexpression of positive regulatory factors,

\section{b}

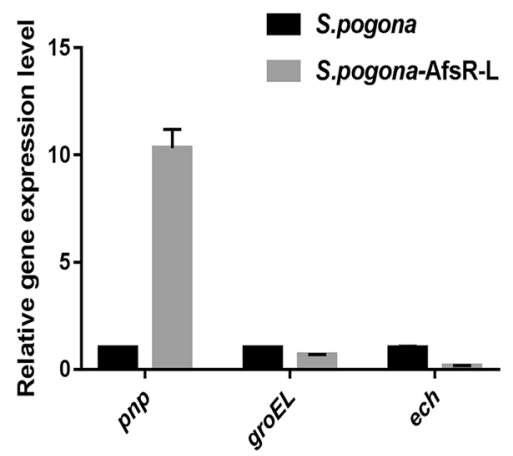

at $120 \mathrm{~h}$. The $16 \mathrm{~S}$ rRNA gene was used as an internal control to quantify the relative expression of target genes. pnp expression level showed an increase, while groEL and ech expression levels were decreased in the mutant. Black bars represent $S$. pogona, and gray bars represent S. pogona-AfsR-L. Gene expression differences were shown by bars height. Error bars represent standard deviation of the mean

knockout of negative regulatory factors, and doubling of secondary metabolic biosynthetic gene clusters ( $\mathrm{Li}$, et al. 2017; Olano, et al. 2008).

Butenyl-spinosyn is a spinosad analogue that is produced by S. pogona. However, the ability of the wild-type $S$. pogona to produce butenyl-spinosyn is low and insufficient for industrial production (Lewer, et al. 2009; $\mathrm{Li}$, et al. 2018). Tang et al. directly cloned $\sim 1.8 \mathrm{~kb}$ fragment containing $s p n S, s p n F$, and $s p n Q$ from spinosad biosynthetic gene clusters and then integrated these

Table 1 Proteins identified from SDS-PAGE gel analysis

\begin{tabular}{|c|c|c|c|c|}
\hline Bands number & Protein description & Gene & MW (KDa) & Possible function \\
\hline A & Polynucleotide phosphorylase & pnp & 79.3 & $\begin{array}{l}\text { Involved in mRNA degradation. Catalyzes } \\
\text { the phosphorolysis of single-stranded } \\
\text { polyribo-nucleotides processively in the } \\
\text { 3'- to 5'-direction. }\end{array}$ \\
\hline $\mathrm{B}$ & $\begin{array}{l}\text { Molecular chaperone } \\
\text { GroEL }\end{array}$ & groEL & 57.2 & $\begin{array}{l}\text { Prevents misfolding and promotes the refolding } \\
\text { and proper assembly of unfolded polypeptides } \\
\text { generated under stress conditions. }\end{array}$ \\
\hline $\mathrm{C}$ & Enoyl-CoA hydratase & ech & 27.5 & $\begin{array}{l}\text { This protein is involved in the pathway fatty acid } \\
\beta \text {-oxidation, which is part of lipid metabolism. }\end{array}$ \\
\hline
\end{tabular}




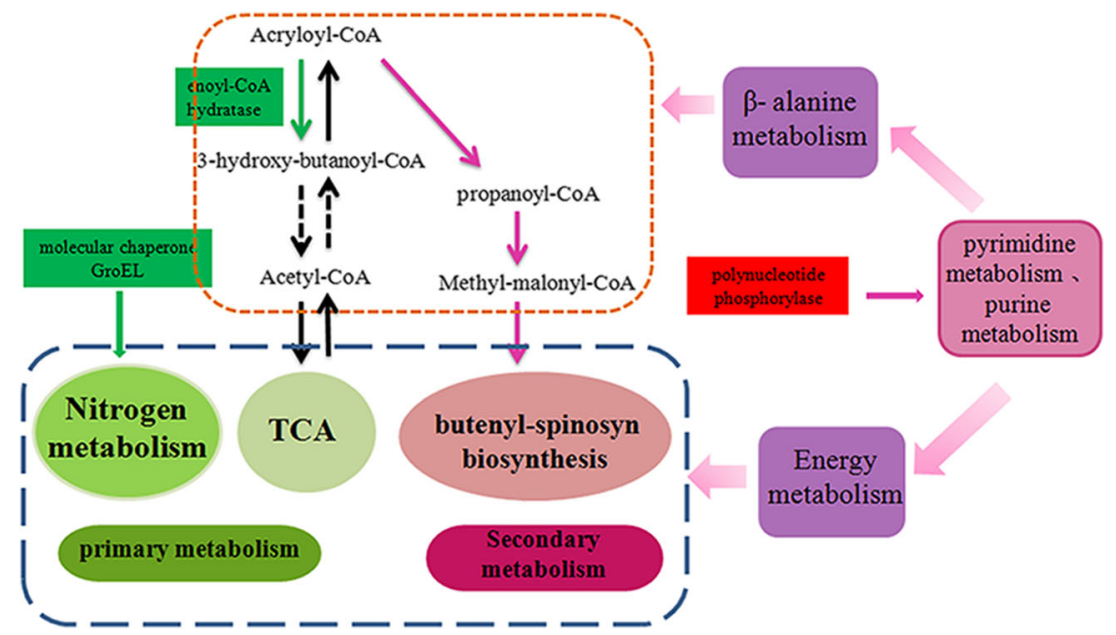

Fig. 6 Regulatory network schematic diagram of afs $R-L$ gene in $S$. pogona. Green rectangle, downregulated protein; red rectangle, upregulated protein; green arrow, downregulatory pathway; mulberry arrow, upregulatory pathway; the downregulated metabolism described in green; the upregulated metabolism described in red or purple. The upregulated pnp gene could directly regulate pyrimidine metabolism and purine metabolism, which strengthened the energy metabolism and $\beta$-alanine metabolism, giving more energy to primary and secondary metabolism and also providing more precursor material to the CoA metabolism pathway. The downregulated groEL gene could reduce the nitrogen metabolism. The downregulated ech gene could generate more methyl-malonyl-CoA, as a key precursor for secondary metabolites synthesis, and reduced the TCA cycle at the same time

phase. This finding showed that AfsR-L affects the biosynthesis of secondary metabolites in S.pogona.

To explore the possible control mechanism of AfsR-L on butenyl-spinosyn production, the differentially expressed proteins of S. pogona-AfsR-L and S. pogona were analyzed and we found that PNPase was upregulated due to the overexpression of AfsRL. It was reported that this protein regulates the amino acid metabolism, organic acid metabolism, cell biosynthesis, and transcription-related enzymes in E. coli (Nurmohamed et al. 2011). Our previous work also found that PNPase affected the growth and the biosynthesis of butenyl-spinosyns in S. pogona, and proved that the PNPase is a key regulatory factor in the metabolism of S. pogona (Li et al. 2018). The analysis of KEGG software reveals that the PNPase directly regulates the pyrimidine and purine metabolism, which produces UDP, CDP, ADP, and GDP that are then catalyzed into UTP, CTP, ATP, and GTP by relevant enzymes, respectively. These compounds participated as energy sources in the cell metabolism, and the UTP and CTP catabolism is $\beta$-alanine, which is further metabolized to generate pyruvate and promote acetyl-COA synthesis. Acetyl-COA is a substrate of butenyl-spinosyn biosynthesis, thus increasing butenyl-spinosyn production. Enoyl-CoA hydrolase catalyzes the formation of 3-hydroxy-butyryl-CoA via acryloyl-CoA and then further generates acetyl-CoA as a raw material for the basic carbon metabolism in the tricarboxylic acid cycle. GroEL affects the assembly and translation of the ribosomal protein factor in the nitrogen metabolism pathway. The biomass of S. pogona-AfsR-L is lower than that of the wild-type strain at the beginning of 5 days of cultivation (Fig. 2). This finding is due to the downregulation of groEL and ech in S. pogona-AfsR-L, which slow down the primary metabolism pathways of $S$. pogona-AfsR-L, such as TCA 
and nitrogen metabolism. The downregulation of ech in S. pogona-AfsR-L decreases the conversion of acryloyl-CoA to acetyl-CoA, enhancing the synthesis of propanoyl-CoA and methyl-malonyl-CoA. In Saccharopolyspora erythaea, methylmalonyl-CoA is a key precursor substance in the erythromycin biosynthetic pathway (Karnicar, et al. 2016). When cultured in a minimal medium, methyl-malonyl-CoA is transformed into succinyl-CoA to participate in the tricarboxylic acid cycle, which decreases the need for the biosynthesis of erythromycin as a precursor (Reeves, et al. 2006). The downregulation of acryloyl-CoA hydrolase in S. pogona-AfsR-L probably increases the conversion of acryloyl-CoA to methyl-malonyl-CoA, providing more precursors for butenyl-spinosyn biosynthesis and promoting the biosynthesis of these secondary metabolites (Fig. 6).

In summary, we first identified the presence of AfsR homolog named as AfsR-L in S. pogona. AfsR-L might function as an important regulatory factor which could render more initial raw materials into the secondary metabolism by decreasing the tricarboxylic acid cycle, nitrogen metabolism, and other primary metabolism, and thus promote the butenyl-spinosyn biosynthesis. At the same time, AfsR-L might indirectly regulate energy and amino acid metabolism, providing more energy and metabolic substances for the growth of $S$. pogona. Our study provided an important target for genetic engineering to improve butenyl-spinosyn biosynthesis in S. pogona.

Author contributions L. L. and S. Y. developed the bacterial recombinant and performed bacterial strain isolation. Y. S., L. G., and J. T. performed HPLC. J. R., X. D., and Z. L. performed LC-MS/MS and data analysis. L. G., H. H., Z. Y., and S. P. performed Total RNA isolation and qRT-PCR analysis. L. G. and H. H. performed Western blot. L. L., H. H., Y. S., and L. X. designed the study and wrote the draft of manuscript. L. G. and Y. S. modified and corrected this manuscript. All authors discussed the results and approved the final manuscript.

Funding information This work was supported by funding from the National Natural Science Foundation of China (31770106), the National Basic Research Program (973) of China (2012CB722301), the Major Research Projects in Hunan Province (2017NK1030), and the Cooperative Innovation Center of Engineering and New Products for Developmental Biology of Hunan Province (20134486).

\section{Compliance with ethical standards}

Conflict of interest The authors declare that they have no conflict of interest.

Research involving human participants and/or animals Ethical approval "All applicable international, national, and/or institutional guidelines for the care and use of animals were followed." All procedures performed in studies involving animals were in accordance with the ethical standards of the Animal Care Committee of Hunan Normal University at which the studies were conducted.

Informed consent Informed consent was obtained from all individual participants included in the study.

\section{References}

Bierman M, Logan R, O’Brien K, Seno ET, Rao RN, Schoner BE (1992) Plasmid cloning vectors for the conjugal transfer of DNA from Escherichia coli to Streptomyces spp. Gene 116:43-49

Bush MJ, Chandra G, Bibb MJ, Findlay KC, Buttner MJ (2016) Genomewide chromatin immunoprecipitation sequencing analysis shows that $w h i B$ is a transcription factor that cocontrols its regulon with whiA to initiate developmental cell division in Streptomyces. mBio 7(2):e00523-00516

Den Hengst CD, Tran NT, Bibb MJ, Chandra G, Leskiw BK, Buttner MJ (2010) Genes essential for morphological development and antibiotic production in Streptomyces coelicolor are targets of BldD during vegetative growth. Mol Microbiol 78(2):361-379

Elliot MA, Locke TR, Galibois CM, Leskiw BK (2003) BldD from Streptomyces coelicoloris a non-essential global regulator that binds its own promoter as a dimer. FEMS Microbiol Lett 225(1):35-40

Engmark M, Jespersen MC, Lomonte B et al (2017) High-density peptide microarray exploration of the antibody response in a rabbit immunized with a neurotoxic venom fraction. Toxicon 138:151-158

Hahn DR, Gustafson G, Waldron C, Bullard B, Jackson JD, Mitchell J (2006) Butenyl-spinosyns, a natural example of genetic engineering of antibiotic biosynthetic genes. J Ind Microbiol Biotechnol 33(2): 94-104

Horinouehi S, Kito M, Nishiyama M, Furuya K, Hug S-K, Miyake K, Beppu T (1990) Primary structure of AfsR, a global regulatory protein for secondary metabolite formation in Streptomyces coelicolor A3(2). Gene (Amsterdam) 95(1):49-56

Huang S, Ding X, Sun Y, Yang Q, Xiao X, Cao Z et al (2012) Proteomic analysis of Bacillus thuringiensis at different growth phases by using an automated online two-dimensional liquid chromatography-tandem mass spectrometry strategy. Appl Environ Microbiol 78(15):5270-5279

Karnicar K, Drobnak I, Petek M, Magdevska V, Horvat J, Vidmar R et al (2016) Integrated omics approaches provide strategies for rapid erythromycin yield increase in Saccharopolyspora erythraea. Microb Cell Factories 15:93

Kim I-K, Lee C-J, Kim M-K, Kim J-M, Kim J-H, Yim H-S et al (2006) Crystal structure of the DNA-binding domain of BldD, a central regulator of aerial mycelium formation in Streptomyces coelicolor A3(2). Mol Microbiol 60(5):1179-1193

Kirst HA (2010) The spinosyn family of insecticides: realizing the potential of natural products research. J Antibiot 63(3):101-111

Lee P-C, Umeyama T, Horinouchi S (2002) afsS is a target of AfsR, a transcriptional factor with ATPase activity that globally controls secondary metabolism in Streptomyces coelicolor A3(2). Mol Microbiol 43(6): 1413-1430

Lewer P, Hahn DR, Karr LL, Duebelbeis DO, Gilbert JR, Crouse GD et al (2009) Discovery of the butenyl-spinosyn insecticides: novel macrolides from the new bacterial strain Saccharopolyspora pogona. Bioorg Med Chem 17(12):4185-4196

Li L, Zheng G, Chen J, Ge M, Jiang W, Lu Y (2017) Multiplexed sitespecific genome engineering for overproducing bioactive secondary metabolites in actinomycetes. Metab Eng 40:80-92

Li L, Rang J, He H et al (2018) Impact on strain growth and butenylspinosyn biosynthesis by overexpression of polynucleotide phosphorylase gene in Saccharopolyspora pogon. Appl Microbiol Biotechnol 102:8011-8021

Liu Y, Sun G, Zhong Z, Ji L, Zhang Y, Zhou J et al (2017) Overexpression of AtEDT1 promotes root elongation and affects medicinal secondary metabolite biosynthesis in roots of transgenic Salvia miltiorrhiza. Protoplasma 254(4):1617-1625

Luo Y, Ding X, Xia L, Huang F, Li W, Huang S et al (2011) Comparative proteomic analysis of Saccharopolyspora spinosa SP06081 and 
PR2 strains reveals the differentially expressed proteins correlated with the increase of spinosad yield. Proteome Sci 9:40

Luo Y, Zhang L, Barton K, Zhao H (2015) Systematic identification of a panel of strong constitutive promoters from Streptomyces albus. ACS Synth Biol 4(9):1001-1010

Maharjan S, Oh TJ, Lee HC, Sohng JK (2008) Heterologous expression of $m e t K 1-s p$ and $a f s R$-sp in Streptomyces venezuelae for the production of pikromycin. Biotechnol Lett 30(9):1621-1626

Millar NS, Denholm I (2007) Nicotinic acetylcholine receptors: targets for commercially important insecticides. Invertebr Neurosci 7(1): $53-66$

Muller S, Pflock M, Schar J, Kennard S, Beier D (2007) Regulation of expression of atypical orphan response regulators of Helicobacter pylori. Microbiol Res 162:1-14

Nurmohamed S, Vincent HA, Titman CM, Chandran V, Pears MR, Du D et al (2011) Polynucleotide phosphorylase activity may be modulated by metabolites in Escherichia coli. J Biol Chem 286(16):1431514323

Olano C, Lombo F, Mendez C, Salas JA (2008) Improving production of bioactive secondary metabolites in actinomycetes by metabolic engineering. Metab Eng 10(5):281-292

Parajuli N, Viet HT, Ishida K, Tong HT, Lee HC, Liou K et al (2005) Identification and characterization of the afsR homologue regulatory gene from Streptomyces peucetius ATCC 27952. Res Microbiol 156(5-6):707-712

Reeves AR, Brikun IA, Cernota WH, Leach BI, Gonzalez MC, Weber JM (2006) Effects of methylmalonyl-CoA mutase gene knockouts on erythromycin production in carbohydrate-based and oil-based fermentations of Saccharopolyspora erythraea. J Ind Microbiol Biotechnol 33(7):600-609

Santos-Beneit F, Rodríguez-García A, Sola-Landa A et al (2009) Crosstalk between two global regulators in Streptomyces: PhoP and AfsR interact in the control of $a f s \mathrm{~S}, p s t \mathrm{~S}$ and phoRP transcription. Mol Microbiol 72(1):53-68

Sun D, Zhu J, Chen Z, Li J, Wen Y (2016) SAV742, a novel AraC-family regulator from Streptomyces avermitilis, controls avermectin biosynthesis, cell growth and development. Sci Rep 6:36915

Tanaka A, Takano Y, Ohnishi Y, Horinouchi S (2007) AfsR recruits RNA polymerase to the afs $\mathrm{S}$ promoter: a model for transcriptional activation by SARPs. J Mol Biol 369:322-333

Tang Y, Xia L, Ding X, Luo Y, Huang F, Jiang Y (2011) Duplication of partial spinosyn biosynthetic gene cluster in Saccharopolyspora spinosa enhances spinosyn production. FEMS Microbiol Lett 325(1):22-29

Tatarko M, Romeo T (2001) Disruption of a global regulatory gene to enhance central carbon flux into phenylalanine biosynthesis in Escherichia coli. Curr Microbiol 43(1):26-32

Virginie Molle WJP, Findlay KC, Buttner MJ (2000) WhiD and WhiB, homologous proteins required for different stages of sporulation in Streptomyces coelicolor A3(2). J Bacteriol 182(5):1286-1295

Wang W, Wang H, Hu H, Peng H, Zhang X (2015) Overexpression of $a f s R$ and optimization of metal chloride to improve lomofungin production in Streptomyces lomondensis S015. J Microbiol Biotechnol 25(5):672-680

$\mathrm{Xu}$ J, Zhang J, Zhuo J et al (2017) Activation and mechanism of a cryptic oviedomycin gene cluster via the disruption of a global regulatory gene, adpA, in Streptomyces ansochromogenes. J Biol Chem 292(48):19708-19720

Yang Q, Ding X, Liu X, Liu S, Sun Y, Yu Z et al (2014) Differential proteomic profiling reveals regulatory proteins and novel links between primary metabolism and spinosad production in Saccharopolyspora spinosa. Microb Cell Factories 13(1):27

Yang Q, Li Y, Yang H, Rang J, Tang S, He L et al (2015) Proteomic insights into metabolic adaptation to deletion of metE in Saccharopolyspora spinosa. Appl Microbiol Biotechnol 99(20): 8629-8641

Yoshihiro Mouri KK, Fujita A, Tezuka T, Ohnishi Y (2017) Regulation of sporangium formation by BldD in the rare actinomycete Actinoplanes missouriensis. J Bacteriol 199(12):e00840-00816

Zhang Y, He H, Liu H, Wang H, Wang X, Xiang W (2016) Characterization of a pathway-specific activator of milbemycin biosynthesis and improved milbemycin production by its overexpression in Streptomyces bingchenggensis. Microb Cell Factories 15(1): 152

Zhu Z, Li H, Yu P, Guo Y, Luo S, Chen Z et al (2017) SlnR is a positive pathway-specific regulator for salinomycin biosynthesis in Streptomyces albus. Appl Microbiol Biotechnol 101(4):1547-1557

Publisher's note Springer Nature remains neutral with regard to jurisdictional claims in published maps and institutional affiliations. 